\title{
Research on Optimization of Linkage Operation of Integrated Energy System in Industrial Parks based on Chaotic Particle Swarm Algorithm
}

\author{
Yang Yang ${ }^{1}$, Mengjin $\mathrm{Hu}^{1}$, Mengju Wei ${ }^{1}$, Yongli Wang ${ }^{2}$, Minhan $\mathrm{Zhou}^{2 *}$ and Yuze $\mathrm{Ma}^{2}$ \\ ${ }^{1}$ Energy Development Research Department, State Grid Hebei Economic Research Institute, Hebei, 050000, China \\ ${ }^{2}$ School of Economics and Management, North China Electric Power University, Beijing,102206, China
}

\begin{abstract}
Industrial parks cover a variety of production capacities and energy-consuming entities, with large load demand and complex energy-using structure, and common problems such as low energy utilization efficiency and unreasonable energy structure. The construction of an integrated energy system (IES) with a combined cooling, heating and power system as the core unit in the industrial park is of great significance for achieving reliable, efficient and clean energy use in the park. Therefore, this article is based on the integrated energy system of the industrial park, aims at the lowest total cost of park operators, and considers the constraints of grid node balance, equipment output and energy storage equipment, and constructs source-grid-load-storage linkage operation optimization model, and build a chaotic particle swarm algorithm (CPSO) to solve the model. Finally, a typical industrial park in my country is taken as an example to analyze the scientificity of the model.
\end{abstract}

\section{Introduction}

Industrial parks cover a variety of production capacities and energy-consuming entities, with large load demand and complex energy-using structure, and common problems such as low energy utilization efficiency and unreasonable energy structure [1-2]. The establishment of an industrial park with cogeneration system as the core unit in the park can realize reliable, efficient and clean energy utilization in the park and friendly interaction between the park and the large power grid. The interactive optimization of the IES of the industrial park can, on the one hand, tap the demand response potential of the participating entities in the park and enhance the benign interaction between users and the grid. On the other hand, it can provide users with better integrated energy supply services [3-4].

Currently, the optimal operation methods of the parklevel IES are diversified. Ref. [5] established a two-level optimization planning model for an IES and an optimization model for system operation scheduling, which are optimized for economy and environmental protection. Ref. [6] proposed an IES optimization planning and operation framework, which considered factors such as technology, economy and environment.

\section{Operation model of multi-energy dispatch links in industrial parks}

\subsection{Objective function}

$$
I_{M}=C_{M}-R_{M}
$$

Where $I_{M}$ is the total cost of the operator; $R_{M}$ is the revenue of the operator; $C_{M}$ is the operating cost of the operator.

$$
C_{M}=\frac{p_{G} Q_{c}}{L \theta \eta}+\sum_{i \in H}\left(\sum_{j=1}^{n} B_{i, j}\right)
$$

Where $Q_{c}$ is the heat supply of the CCHP unit; $p_{G}$ is the gas price; $L$ is the low heating value of the gas; $\theta$ is the heat-to-electricity ratio of the CCHP unit; $\eta$ is the power generation efficiency of the CCHP unit.

$$
p_{i}=\frac{\eta h_{i}}{\left(1-\eta-\eta_{1}\right) \delta_{h}}
$$

Where $p_{i}$ is the power generation of CCHP during the time period $i ; h_{i}$ is the heat generation power of the CCHP during the time period $i$, which also equal to the 
sum of the heat loads of all users in the industrial park; $\eta_{1}$ is the heat loss coefficient; $\delta_{h}$ is the heat coefficient.

\subsection{Restrictions}

1) Node power balance

$$
\left\{\begin{array}{l}
P_{i, S}-P_{i, D}-U_{i} \sum_{j \in i} U_{j}\left(G_{i j} \cos \theta_{i j}+B_{i j} \sin \theta_{i j}\right)=0 \\
Q_{i, S}-Q_{i, D}-U_{i} \sum_{j \in i} U_{j}\left(G_{i j} \sin \theta_{i j}-B_{i j} \cos \theta_{i j}\right)=0
\end{array}\right.
$$

Where $P_{i, S}, Q_{i, S}$ respectively represent the active and reactive power generation of the grid node $i$; $P_{i, D}, Q_{i, D}$ respectively represent the active and reactive power of the load connected to the node $i ; U_{i}$ represents the voltage amplitude of the node $i ; \theta_{i j}$ represents the voltage angle difference between the node $i$ and the node $j ; G_{i j}, B_{i j}$ respectively are the node admittance The real and imaginary parts of the matrix.

2) Upper and lower limits of power output

$$
\left\{\begin{array}{l}
P_{s}^{\min } \leq P_{s} \leq P_{s}^{\max } \\
Q_{s}^{\min } \leq Q_{s} \leq Q_{s}^{\max }
\end{array}\right.
$$

Where $P_{S}^{\max }, P_{S}^{\min }$ respectively represent the upper and lower limits of the equivalent power output active power; $Q_{S}^{\max }, Q_{S}^{\min }$ respectively represent the upper and lower limits of the equivalent power output reactive power.

3) Equipment output and climb rate constraints

$$
\left\{\begin{array}{l}
P_{i, t, \min } \leq P_{i, t} \leq P_{i, t, \max } \\
P_{f, t, \min } \leq P_{f, t} \leq P_{f, t, \max }
\end{array}\right.
$$

Where $P_{i, t, \text { min }}$ and $P_{i, t, \text { min }}$ are the maximum and minimum output of unschedulable equipment; $P_{f, t, \max }$ and $P_{f, t, \text { min }}$ are the effective dispatching intervals of dispatchable units.

4) Energy storage battery constraints

$$
\left\{\begin{array}{l}
S O C_{\min } \leq S O C(t) \leq S O C_{\max } \\
P_{c h_{-} e, \max } \leq P_{b a t}(t) \leq P_{d i s_{-} e, \max } \\
P_{c h_{-} e}(t) \times P_{d_{\text {dis_e }} e}(t)=0
\end{array}\right.
$$

Where $S O C(t), S O C\left(t_{0}\right)$ are the remaining capacity of the energy storage battery at $t$ and $t_{0}$ respectively; $\delta$ is the self-discharge rate of the energy storage battery, $\% / \mathrm{h} ; S O C_{\min }$ and $S O C_{\max }$ the minimum and maximum constraints of the remaining capacity; $P_{c h \_e, \max }$ and $P_{\text {dis_e } \max }$ are the maximum charge and discharge power.

\subsection{Solving Algorithm}

The specific process of the CPSO is as follows:

Step 1: Initialize and set the parameters and the maximum number of iterations in the algorithm.

Step 2: Use the chaotic sequence to initialize its position and velocity.

Step 3: If the fitness value of the particle is better than $p_{\text {best }}$, the latest position is recorded as $p_{\text {best }}$.

Step 4: Update the position and velocity of the particles.

Step 5: Calculate the fitness value of each feasible solution obtained by chaos optimization, and find the best feasible solution among all feasible solutions.

Step 6: Replace the positions of all particles in space.

Step 7: If the maximum number of cycles is reached, stop searching and get the global optimal solution, otherwise continue to step 3 .

\section{Case analysis}

\subsection{Basic Data}

Based on the data of load and natural resources in an industrial park, this paper conducts simulations to install fans, photovoltaics, CCHP systems, electric boilers (EB), double-mode refrigeration units (DC), energy storage (ES), ground source heat pumps (GSHP), and conventional refrigeration units (CR) for the park. In this case, the power grid of the industrial park has a strong power supply regulation capability, which can reduce abandonment of wind and light, and use the price difference to reduce electricity costs. The IES sourcegrid-load-storage linkage operation optimization model proposed in this paper is used to optimize the integrated energy system scheduling of industrial parks to achieve the highest requirements for operators' profits.

The use price of energy is an important factor that affects the operation results. The electricity price in the park adopts the time-of-use electricity price, the DC implements the double-storage electricity price, and the gas price adopts the fixed gas price. The energy price curve is shown in Figure 1. 


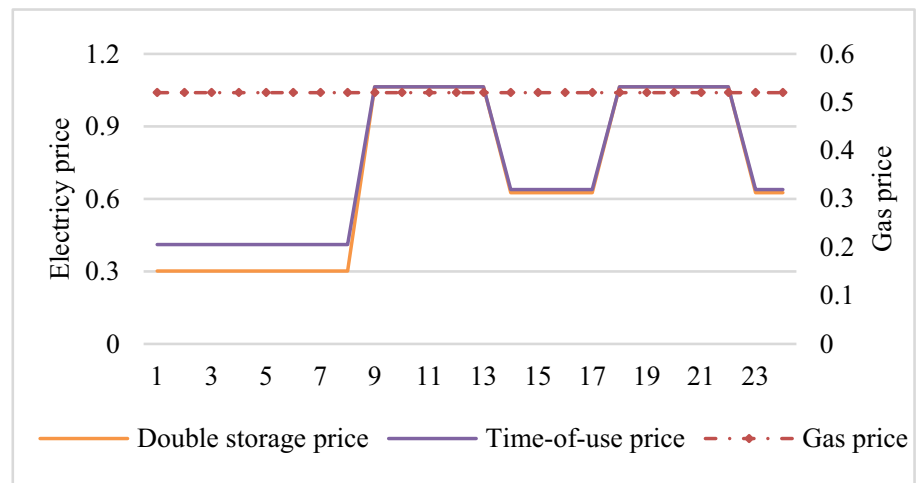

Figure 1. Energy price curve

\subsection{Results and analysis}

Compare CPSO, particle swarm algorithm (PSO) and genetic algorithm (GA). TABLE I shows the solution results of each algorithm. It can be seen that the CPSO has higher calculation efficiency and better calculation results.

Table 1. Solving results of each algorithm

\begin{tabular}{cccc}
\hline Algorithm & Iterations & Calculation time (s) & Cost (yuan) \\
\hline CPSO & 100 & 113.92 & 7937.02 \\
Particle Swarm & 100 & 128.21 & 8103.74 \\
Algorithm & 100 & 133.06 & 8181.27 \\
\hline
\end{tabular}

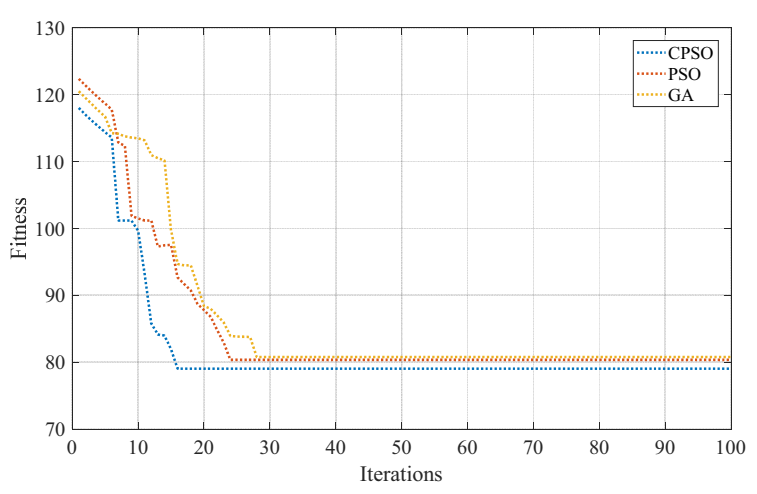

Figure 2. Comparison of CPSO, PSO and GA

The power load curve of the industrial park before and after linkage optimization is shown in Figure 3. It can be seen from Figure 3 that the time limit for the electric load before the interaction is 6:00-18:00, and peak clipping should be performed at these times. The model constructed in this paper keeps the shape of the original load curve as much as possible during the nonover-limit period, so as to avoid bringing users too much power plan adjustment. In addition, the calculation

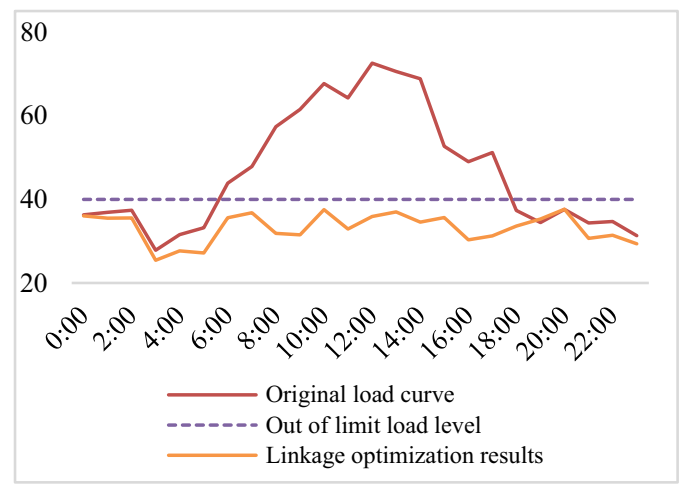

Figure 3. The power load curve of the industrial park before and after optimization

results show that in order to achieve the interactive effect shown in Figure 4 for the traditional interactive method, the operator needs to give higher peak-shaving compensation electricity prices during all interactive periods. Therefore, the optimization model constructed in this paper can reduce the economic costs that operators pay for linkage. The equipment output of the optimized heating and cooling systems are shown in Figure 4 and Figure 5, respectively. 


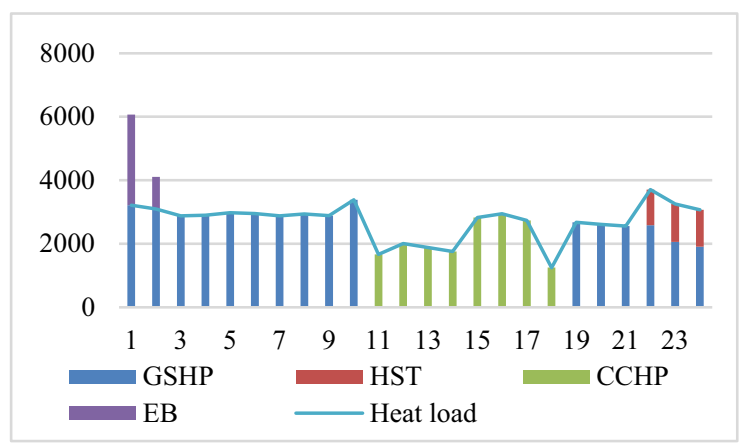

Figure 4. The output of the heating system in the industrial park after optimization

\section{Conclusion}

Under the framework of the integrated energy system of the industrial park, an optimal scheduling model for multi-link operation of source, network, load and storage, and the corresponding solution method are proposed, and the influence of multiple energy synergies is analyzed. Finally, the applicability of the proposed method in the optimal scheduling of the integrated energy system of the industrial park is verified, and it has a fast solution speed and the best overall result. The next step will be to solve the problem of how to quickly solve non-convex models for multi-objective optimization.

\section{Acknowledgments}

This paper is supported by "Key R \& D Program Project of Hebei Province (19216109D)" and "Major supporting projects in Hebei (SGHEJY00NYJS2000055)”.

\section{References}

1. Liu Dichen, Ma Hengrui, Wang Bo, Gao Wenzhong, Wang Jun, Yan Bingke.(2018) Operation optimization of regional integrated energy system

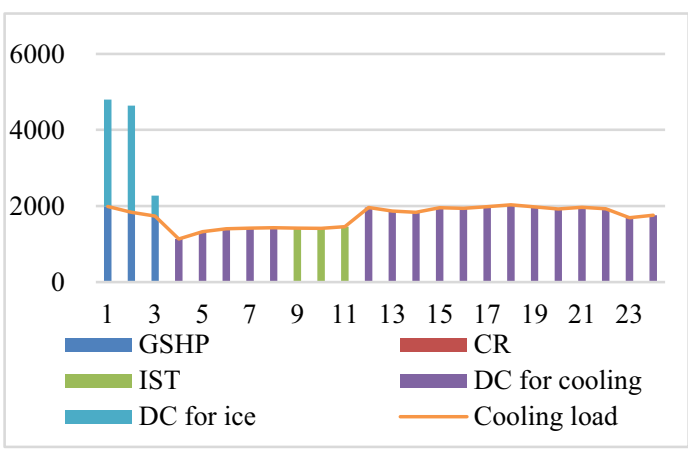

Figure 5. The output of the cooling system in the industrial park after optimization

with combined cooling, heating and power and energy storage. Automation of Electric Power Systems. 42: 113-120+141.

2. Wu Fubao, Liu Xiaofeng, Sun Yimei, Chen Ning, Yuan Tiejiang, Gao Bingtuan. (2018) Multi-park game optimization strategy based on combined cooling, heating and power. Automation of Electric Power Systems, 42: 68-75.

3. Jiang Ziqing, Hao Ran, Ai Qian. (2017) Research on the interaction mechanism of industrial parks based on multi-energy complementation of cooling, heating and power". Electric Power Automation Equipment. 37: 260-267.

4. Zhang Qin, Wang Xifan, Fu Min, Wang Jianxue.(2009) Smart grid from the perspective of demand response". Automation of Electric Power Systems, 33: 49-55.

5. Yu Bo, Sun Hengnan, Xiang Tianchun, Zhang Peng. (2016) Integrated energy system planning and design method". Electric Power Construction, 37: 78-84.

6. Li Jinghua, Sang Chuanchuan. (2015) Energy comprehensive system optimization planning and operation framework". Electric Power Construction, 36: 41-48. 